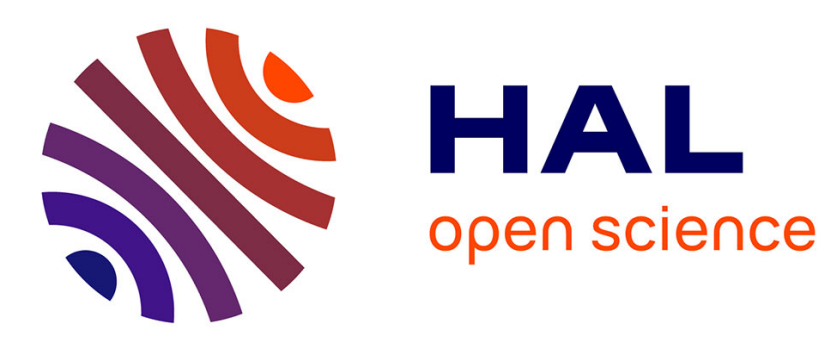

\title{
DES FEMMES CHEZ LES SAPEURS-POMPIERS
}

Roland Pfefferkorn

\section{To cite this version:}

Roland Pfefferkorn. DES FEMMES CHEZ LES SAPEURS-POMPIERS. Cahiers du Genre, 2006, 40, pp.203-230. 10.3917/cdge.040.0203 . hal-01292032

\section{HAL Id: hal-01292032 \\ https://hal.science/hal-01292032}

Submitted on 22 Mar 2016

HAL is a multi-disciplinary open access archive for the deposit and dissemination of scientific research documents, whether they are published or not. The documents may come from teaching and research institutions in France or abroad, or from public or private research centers.
L'archive ouverte pluridisciplinaire HAL, est destinée au dépôt et à la diffusion de documents scientifiques de niveau recherche, publiés ou non, émanant des établissements d'enseignement et de recherche français ou étrangers, des laboratoires publics ou privés. 


\section{DES FEMMES CHEZ LES SAPEURS-POMPIERS}

\section{Roland Pfefferkorn}

\section{L'Harmattan | « Cahiers du Genre »}

2006/1 n 40 | pages 203 à 230

ISSN 1298-6046

ISBN 9782296005012

Article disponible en ligne à l'adresse :

http://www.cairn.info/revue-cahiers-du-genre-2006-1-page-203.htm

\section{Pour citer cet article :}

Roland Pfefferkorn, «Des femmes chez les sapeurs-pompiers », Cahiers du Genre 2006/1 ( ${ }^{\circ}$ 40), p. 203-230.

DOI 10.3917/cdge.040.0203

Distribution électronique Cairn.info pour L'Harmattan.

(C) L'Harmattan. Tous droits réservés pour tous pays.

La reproduction ou représentation de cet article, notamment par photocopie, n'est autorisée que dans les limites des conditions générales d'utilisation du site ou, le cas échéant, des conditions générales de la licence souscrite par votre établissement. Toute autre reproduction ou représentation, en tout ou partie, sous quelque forme et de quelque manière que ce soit, est interdite sauf accord préalable et écrit de l'éditeur, en dehors des cas prévus par la législation en vigueur en France. Il est précisé que son stockage dans une base de données est également interdit. 


\title{
Des femmes chez les sapeurs-pompiers
}

\section{Roland Pfefferkorn}

\begin{abstract}
Résumé
Cet article est consacré à l'arrivée des femmes dans les corps de sapeurs-pompiers. Si ces corps se sont progressivement ouverts depuis trente ans, ils ne sont pas devenus pour autant des univers «mixtes». Davantage encore que l'armée ou la police, ils restent marqués par une forte hégémonie masculine. Les jeunes femmes qui entrent dans ce milieu doivent faire face à de multiples réactions défensives de la part de leurs collègues masculins. La répartition des tâches est structurée suivant la hiérarchie des rapports sociaux de sexe. Le contenu des emplois varie suivant le sexe. La division du travail traditionnelle entre les sexes y est reproduite. La puissance des stéréotypes de sexe apparaît presque à l'état brut. Cet exemple permet de mesurer à quel point l'entrée des femmes dans un milieu d'hommes ne va vraiment pas de soi.
\end{abstract}

FÉMINISATION DES PROFESSIONS - SAPEURS-POMPIERS - FÉMINITÉ MASCULINITÉ — VIRILITÉ — IDENTITÉ MASCULINE — COLLECTIF DE TRAVAIL

L'arrivée des femmes dans les corps de sapeurs-pompiers est relativement récente. Comme d'autres milieux professionnels qui se sont féminisés au cours des dernières décennies ${ }^{1}$, ces corps se sont progressivement ouverts depuis trente ans. Mais ils ne sont pas devenus pour autant des univers «mixtes ». Ils restent marqués par une forte hégémonie masculine. Hégémonie quanti-

\footnotetext{
${ }^{1}$ Cf. notamment Appert et al. (1983), Gadrey (2001), Bihr et Pfefferkorn (1996, 2002), Hirata et Senotier (1996), Maruani (1998) et Fortino (1999, 2002).
} 
tative d'abord : en 2003 les femmes représentent à peine 6 \% de l'ensemble des sapeurs-pompiers (volontaires et professionnels). Cette part reste inférieure à $1 \%$ parmi les professionnels : on y compte en effet moins d'une femme sur deux cents.

En comparaison avec ces autres "bastions masculins" qu'étaient traditionnellement l'armée ou la police (Reynaud 1988 ; Sorin 2003 ; Monrique 2004 ; Pruvost 2005), l’ouverture des corps de sapeurs-pompiers est à la fois moindre et moins rapide. Au sein de l'armée, les effectifs féminins sont passés de $7,1 \%$ en 1992 à $11,4 \%$ en $2002^{2}$. Dans la police, il y a désormais $14 \%$ de femmes en service actif. Cette proportion augmente régulièrement et, malgré les obstacles, quels que soient les niveaux et les grades, rares sont les promotions récentes comportant moins d'un tiers de femmes ${ }^{3}$. Sur le plan quantitatif, la part des femmes dans les corps de sapeurs-pompiers professionnels reste donc particulièrement faible au regard des chiffres observés dans l'armée ou la police. Ces secteurs ont amorcé leur féminisation un peu plus tôt, mais surtout plus vite et bien davantage. La féminisation y a commencé par les grades intermédiaires et supérieurs, ce qui a permis plus facilement d'aller à l'encontre de certains comportements machistes (Bouchard 1996). Par ailleurs, il y avait d'emblée la volonté d'imposer un seuil minimal de femmes, par exemple sur les navires militaires, afin d'assurer l'intégration des femmes embarquées dans des conditions acceptables (Dufoulon et al. 1998 ; Trompette et al. 1999).

\footnotetext{
${ }^{2}$ En 2002 les femmes représentent 46,3 \% des services de santé, 18,2 \% de l'armée de l'air, $12,1 \%$ des effectifs de la marine, $11,4 \%$ de la gendarmerie et $9,5 \%$ de l'armée de terre. Tout récemment, après la suppression du service national, la progression a été particulièrement forte parmi les volontaires recrutés pour remplacer les appelés : la part des femmes dans cette catégorie est passée de 2 \% en 1999 à 26,6 \% en 2002 (Monrique 2004). Même dans la marine militaire embarquée, qui s’est féminisée tardivement (à partir de 1993), la part des femmes se situe à un niveau nettement plus élevé que dans les corps de sapeurs-pompiers professionnels : 4,4\% des marins embarqués sont des femmes. Actuellement douze navires ont un équipage mixte, ce nombre va augmenter prochainement. Par ailleurs, tous les navires mixtes comportent $10 \%$ de femmes depuis le début et leur part doit passer prochainement à $15 \%$ (Dufoulon et al. 1998 ; Trompette et al. 1999 ; Monrique 2004).

${ }^{3}$ Par contre, les femmes sont largement majoritaires dans les services administratifs de la police où elles occupent $73 \%$ des postes, ce qui explique qu'elles représentent $22 \%$ des effectifs globaux (Pruvost 2005).
} 
Dans l'univers des sapeurs-pompiers, cette hégémonie masculine se manifeste aussi à un autre niveau, tant sur le plan des discours que des pratiques. L'arrivée des femmes suscite en effet toujours des réactions masculines de rejet particulièrement vives près de trente ans après l'entrée des premières femmes. Les hommes se vivent comme des «communautés » fortement construites sur une opposition de genre où la « caserne » constitue l'envers masculin d'un univers familial fantasmé comme féminin. Les stéréotypes sociaux de sexe y sont particulièrement développés, davantage peut-être que dans l'armée ou dans la police, en raison précisément de la place plus réduite qu'y occupent les femmes. Face à la lente féminisation de leur profession, les hommes sapeurs-pompiers mettent en œuvre diverses pratiques individuelles et collectives de défense qui toutes visent à rejeter les femmes, ou du moins à les enfermer dans un statut et des fonctions subalternes.

\section{Une entrée modeste d'《 héritières " dans un monde d'hommes mythifié}

Ce n’est qu'au milieu des années 1990, alors que les femmes représentaient encore moins de $2 \%$ des effectifs totaux, que le monde des sapeurs-pompiers commence lentement à prendre conscience de leur arrivée. Les cercles dirigeants nationaux semblent alors souhaiter l'incorporation de femmes dans ce milieu masculin plutôt hostile. En octobre 1996, le mensuel de la Fédération nationale des sapeurs-pompiers de France (FNSPF), Sapeur-Pompier Magazine, consacre un article aux femmes sapeurs-pompiers. Prenant à contre-pied les préjugés du milieu, l'auteur insiste sur l'ancienneté de la présence des femmes dans le combat contre les incendies. Il remarque que dès le Moyen Âge les municipalités faisaient obligation aux femmes de participer à la lutte contre le feu en formant la chaîne des seaux, sous peine d'amende, voire de prison ${ }^{4}$. Et de citer l'exemple de Lille, où un ban du 15 juin 1383, confirmé en 1397, précise :

\footnotetext{
${ }^{4}$ Jean Deville, « Des sapeurs-pompiers féminins », Sapeur-Pompier Magazine, octobre 1996.
} 
Quand la cloche sonnera, incontinent, ceux et celles, tant hommes que femmes, iront pour aider au feu.

La présence de femmes est attestée aussi au $\mathrm{XV}^{\mathrm{e}}$ ou au $\mathrm{XVI}^{\mathrm{e}}$ siècle à Amiens, à Douai, à Besançon, à Montbéliard ou à Toulouse. Leur participation à la lutte contre les incendies en ces temps reculés est mobilisée implicitement pour contrer les arguments de ceux qui s'opposent à leur arrivée récente au sein des corps de sapeurs-pompiers. L'argument de l'ancienneté de la présence de femmes dans la lutte contre les incendies est cependant équivoque, car toutes les femmes n'étaient pas réquisitionnées dans le passé. La corvée visait en fait d'abord les prostituées ${ }^{5}$. Par ailleurs, s'il est vrai que les femmes participaient aux chaînes d'eau lors de la lutte contre les incendies, elles étaient généralement chargées de ramener les seaux vides, les hommes faisant la chaîne montante.

L'entrée des femmes dans les corps de sapeurs-pompiers volontaires ou professionnels remonte maintenant à une trentaine d'années. Elle est rendue possible explicitement par le décret $\mathrm{n}^{\circ}$ 76-1007 du 25 octobre 1976 qui ajoute à l'article 9 du décret du 7 mars 1953 un alinéa $1^{\mathrm{er}}$ :

Les corps de sapeurs-pompiers communaux peuvent être composés de personnels tant masculins que féminins.

La circulaire du 15 novembre 1976 ( $\left.{ }^{\circ} 76-524\right)$ rappelle cependant que la présence féminine en marge des corps de sapeurs-pompiers volontaires est antérieure :

Des filles et des épouses de sapeurs-pompiers participent déjà au relevage et au transport des blessés de la route et interviennent parfois pour assurer le premier départ de véhicules de lutte contre l'incendie.

Les femmes n'étaient donc pas totalement extérieures au milieu des sapeurs-pompiers volontaires. Leur participation informelle aux interventions précédait donc leur admission officielle dans les unités. Cette circulaire précise en outre que les femmes ne doivent pas être confinées de manière permanente dans des tâches exclusivement administratives, mais exercer

\footnotetext{
${ }^{5}$ Les femmes concernées à Amiens étaient les «filles de joye », à Douai les « filles des deux rues », à Toulouse les «filles du monde », à Besançon les « filles communes » (Jean Deville, op. cit.).
} 
l'ensemble des fonctions revenant statutairement à tout sapeurpompier. L'adoption de ces mesures en 1976 s'explique aussi, conjoncturellement, par les controverses qui ont suivi l'arrivée, sans cadre légal explicite, des premières femmes dans quelques casernes au cours des années précédentes.

L'année suivante les premières femmes seront recrutées officiellement. Le $1^{\mathrm{er}}$ février 1977, le corps départemental de Seineet-Marne recrute sa première femme pompier volontaire et quelques mois plus tard sa première femme pompier professionnelle. Cette même année 1977, dans la Meuse, c'est l'épouse du lieutenant commandant le centre de secours qui devient la première femme pompier du département. Dorénavant les femmes peuvent entrer dans les corps civils, volontaires et professionnels, mais cette arrivée se fera longtemps au compte-goutte. Si, deux ans plus tard, en 1978, plus de la moitié des départements ont engagé quelques femmes dans les corps de sapeurs-pompiers, leur nombre reste très limité. Pour la France entière on en compte à peine 165, dont 141 volontaires. Quinze ans plus tard, à la fin de l'année 1991, l'effectif féminin total atteint le chiffre de 3 637, dont 120 seulement parmi les professionnels. La proportion de femmes se situe toujours à un niveau très modeste : moins de $2 \%$ globalement. Ce fait explique partiellement l'absence d'interrogation sur l'arrivée des femmes sapeurspompiers dans une recherche menée en Loire-Atlantique au début des années 1990 (Retière 1994). Pourtant, dans la mesure où ce sont les changements intervenus dans les corps de sapeurspompiers volontaires qui étaient au centre de la recherche, ce « silence » sur les femmes (Perrot 1998), alors que la féminisation était amorcée, surprend malgré tout. Une dizaine d'années plus tard, en 2002, le chiffre de 15000 femmes sapeurs-pompiers est dépassé, soit, en chiffre absolu, un peu plus que le nombre de femmes en service actif dans la police (Pruvost 2005). Mais en proportion, leur part est nettement plus réduite : $6 \%$ des effectifs parmi l'ensemble des sapeurs-pompiers civils ${ }^{6}$ et $0,5 \%$

\footnotetext{
${ }^{6}$ Au total, il y a 236689 sapeurs-pompiers civils (volontaires et professionnels) en 2002 auxquels il faut ajouter les corps de sapeurs-pompiers professionnels militaires. Il faudra attendre l'année 2000 pour voir les 35 premières femmes intégrer les corps militaires localisés essentiellement à Paris et dans les dépar-
} 
seulement parmi les professionnels. Elles sont à peine six professionnelles à Nice ou dix à Strasbourg.

Les corps de sapeurs-pompiers volontaires intègrent la plupart des nouveaux venus, y compris ceux qui envisagent d'entrer par la suite dans les sapeurs-pompiers professionnels. Ils regroupent $85 \%$ des pompiers civils. Leurs membres exercent une autre profession, mais leur activité n'est pas pour autant totalement bénévole. Outre les rétributions symboliques, la reconnaissance sociale se traduit aussi par des prestations (des vacations) dont le montant a sensiblement augmenté ces dernières décennies. Par ailleurs, les sapeurs-pompiers volontaires bénéficient de possibilités, certes limitées mais réelles, de mobilité sociale ascendante "à l'intérieur d'un champ de destins qui ne dépasse guère l'horizon du monde populaire. Mais rien ne serait plus trompeur que de la sous-estimer » (Retière 1994, p. 98). Enfin, ils ont droit sous certaines conditions à une retraite complémentaire de 1800 euros par an ${ }^{7}$.

Précisons encore qu'à l'image de leurs aînés, aujourd'hui comme hier (Lussier 1987 ; Kihl 2000), les volontaires de moins de trente ans sont très typés sociologiquement: ils viennent d'abord des milieux populaires (ouvriers, employés ou agriculteurs), tandis que les enfants de cadres et professions intellectuelles supérieures sont relativement peu représentés ${ }^{8}$. Notons cependant que dans un passé encore récent les membres des catégories moyennes et supérieures salariées ou des professions libérales

tements limitrophes et à Marseille. Les militaires représentent moins de $4 \%$ de l'ensemble des sapeurs-pompiers, mais plus de $20 \%$ des professionnels.

${ }^{7}$ Des vacations sont versées aux sapeurs-pompiers volontaires à la fois pour la durée des formations et des manœuvres, les "gardes », et pour les interventions elles-mêmes. Comme le remarquent des chercheurs rennais : «Être volontaire » peut devenir un job d'été ou un " petit boulot » d'étudiant (cf. la synthèse d'une étude sociologique réalisée par des chercheurs rennais portant sur les volontaires de moins de 30 ans, publiée dans Sapeur-Pompier Magazine, mai 2002). La création d'une retraite complémentaire a été annoncée par le ministre de l'Intérieur lors du congrès de la FNSPF le 27 septembre 2003.

${ }^{8}$ La situation des sapeurs-pompiers volontaires en France. Bilan de l'enquête nationale réalisée en 1997 et 1998, Observatoire national du volontariat dans les corps de sapeurs-pompiers, ministère de l'Intérieur, Direction de la défense et de la sécurité civile, septembre 1998, p. 18-19. 
étaient purement et simplement absents des corps de sapeurspompiers volontaires (Retière 1994).

Suivant la classification utilisée ailleurs par Danièle HervieuLéger (1999), on peut regrouper les sapeurs-pompiers en deux grands idéaux types: les "héritiers " et les " convertis ». Les premiers sont plutôt des fils et des filles de sapeurs-pompiers issus d'une lignée. Être pompier fait alors partie de leur identité et de leur histoire, la référence centrale est plutôt le père et l'ancrage local est fort. On peut assimiler à ce premier groupe le cas des hommes et - plus rarement - des femmes qui intègrent un corps de volontaires dans la continuité de la sociabilité locale. Le rôle des pairs ou, par exemple, de l'association sportive est alors déterminante dans la décision d'entrer dans le corps de sapeurs-pompiers volontaires. Le second idéal type regroupe ceux qui sont devenus pompiers par aspiration. Ils se vivent davantage comme "des techniciens du risque ", la compétence et la performance professionnelle priment alors sur le dévouement, la vocation ou l'ancrage dans un lieu (Retière 1994). La référence centrale est alors davantage le pompier professionnel. Mais, comme les membres de ce groupe ne bénéficient pas d' " héritage ", ils doivent faire l'apprentissage non seulement d'un métier, mais aussi d'une culture et s'insérer dans un collectif qui leur est en partie étranger. Cette intégration au sein d'un corps de volontaires ne constitue cependant pas l'objectif final d'une fraction non négligeable des membres de ce second groupe, dans la mesure où leur aspiration profonde consiste à passer du volontariat au statut de sapeur-pompier professionnel.

Encore très minoritaires, les femmes qui entrent dans le milieu sont de très loin d'abord des " héritières ». On observe un phénomène analogue dans l'armée ou la police (Monrique 2004 ; Pruvost 2005), mais aussi dans la vie politique (Criqui 1992). Déjà initiées, l'intégration semble alors a priori plus facile pour elles. Les " converties » sont nettement moins nombreuses, la valorisation médiatique de la présence de femmes dans les corps de sapeurs-pompiers, à partir du milieu des 
années 1990, semble avoir joué un rôle dans leur conversion récente ${ }^{9}$.

Quoique encore lente, l'augmentation de la part des femmes parmi les pompiers volontaires est plus rapide cependant que parmi les professionnels. Elle est directement liée aux difficultés chroniques croissantes de recrutement. Les "vocations » se raréfient en effet aussi dans ce secteur. Traditionnellement, jusqu'aux années 1970, l'entrée dans les corps de sapeurspompiers volontaires intervenait généralement après le service militaire et rarement avant 25 ans. Au cours des dernières décennies, l'âge d'entrée a eu tendance à baisser au-dessous de 20 ans, c'est-à-dire qu'un nombre croissant de jeunes entrent désormais dans les corps de sapeurs-pompiers au cours de leurs études. Récemment, l'âge minimum a même été abaissé à 16 ans. Ces nouvelles recrues rencontrent des problèmes de disponibilité directement liés aux contraintes découlant de l'éloignement des lieux des études ou des emplois. Le turn-over les touche beaucoup plus souvent. Il est avant tout lié à la poursuite des études ou à l'entrée dans une activité professionnelle impliquant un changement de domicile. De plus, les plus motivés des jeunes pompiers volontaires ont tendance à terme à quitter le corps d'origine pour rejoindre une affectation dans un corps de professionnels, civil ou militaire ${ }^{10}$.

Les tâches des sapeurs-pompiers volontaires se sont fortement diversifiées au cours des dernières années. Le champ des interventions est à l'heure actuelle particulièrement large. Contrairement à des représentations récurrentes, alimentées par l'ampleur des incendies estivaux de forêts et largement entretenues par les médias et par les pompiers eux-mêmes, leurs tâches ne se limitent pas à la lutte contre les incendies, loin de là.

\footnotetext{
${ }^{9}$ On observe également un tel phénomène de surmédiatisation dans le cas des femmes policières (Pruvost 2005).

${ }^{10}$ Le rapport de mission Sapeurs-Pompiers : état des lieux et réflexion prospective sur le volontariat (mars 2003), confié à Jean-Paul Fournier par le ministre de l'Intérieur, met en relief l'importance de la crise du volontariat chez les sapeurs-pompiers. Le turn-over est actuellement de l'ordre de $10 \%$ par an. La décision, annoncée lors du congrès de la FNSPF le 27 septembre 2003, d'abaisser à 16 ans l'âge minimum pour l'entrée dans les corps de sapeurspompiers volontaires vise précisément à répondre à cette crise du volontariat.
} 
La lutte contre le feu représente seulement $6 \%$ de l'ensemble des interventions, les sapeurs-pompiers sont davantage mobilisés par toute une série d'autres tâches : les accidents de circulation, les conséquences des intempéries ou de la canicule, les catastrophes technologiques, les accidents de santé, la capture d’animaux familiers ou exotiques, etc. (Géo 15 mai 2002).

Par contre, le thème de la lutte contre le feu inspire toujours les représentations dominantes. Par exemple, les discours officiels ou les articles de presse multiplient la référence aux « soldats du feu » et cultivent systématiquement les métaphores guerrières ${ }^{11}$. Certes, la présence des femmes est prise en compte et certains médias titrent désormais aussi sur « les combattantes du feu » ou « les femmes du feu ». Ces représentations mythiques donnent cependant une image très largement biaisée par rapport à la réalité des interventions des sapeurs-pompiers. Ces métaphores contribuent à la fois à l'héroïsation du statut de sapeur-pompier et à l'affirmation de valeurs viriles propres à ce milieu d'hommes. La lutte contre le feu n'est-elle pas, aux yeux de certains, "porteuse de symboles et de valeurs psychosexuelles [sic] " une lutte qui « devrait être réservée aux hommes, protecteurs virils du groupe » (Deville, op. cit.) ?

\section{Des réactions défensives à l'arrivée des femmes}

L'entrée des femmes dans les corps de sapeurs-pompiers suscite des réactions défensives fortes de la part des hommes ${ }^{12}$. Certes, la moitié des hommes interrogés ${ }^{13}$ tient dans un premier

${ }^{11}$ Le congrès 2003 de la FNSPF en a été un bon révélateur. Le slogan de la page d'accueil du site officiel du congrès : "Plein feu sur les pompiers » donne le ton, mais tant le discours tenu par le ministre de l'Intérieur à cette occasion que les comptes rendus dans la presse ont mis l'accent de manière centrale sur la figure du pompier luttant contre le feu.

12 À l'inverse, il est frappant de constater que les hommes qui entrent dans les professions fortement féminisées (par exemple celle d'infirmière) n’ont pas à faire face à ce type de réactions défensives, ils sont plutôt bien accueillis (Kergoat et al. 1992).

${ }^{13}$ La quasi-totalité des entretiens ont été réalisés durant l'année 2002/2003 par un groupe d'étudiant(e)s de la Faculté des sciences sociales de l'université Marc Bloch de Strasbourg, dans le cadre des enquêtes pédagogiques prévues dans leur cursus. Qu’Adeline Gilson, Sophie Meder, Cathia Papi, Adeline Schreiner, 
temps un propos assez consensuel et semble accepter la présence parmi eux de femmes. Par contre d'autres réagissent d'emblée de manière négative et n'hésitent pas à stigmatiser ces dernières. Dans le premier groupe, on trouve la plupart des membres de la hiérarchie et les femmes elles-mêmes. Elles insistent sur la dimension professionnelle de leur activité et sur le caractère quasi asexué du groupe :

Ici, il n'y a pas de filles, il n'y a pas de garçons, ce n'est que des pompiers [...]. On a tous le même travail, les mêmes choses à faire [...]. Les formations et les manœuvres sont les mêmes...

La hiérarchie adhère aussi à ce type de discours qui met l'accent à la fois sur les compétences professionnelles et sur l'adhésion des femmes à l'esprit de corps. On retrouve ce type d'argumentation dans la hiérarchie policière ou militaire. Par exemple, le capitaine de vaisseau, commandant la frégate Montcalm, qui a un équipage mixte depuis 1993, fait cette observation :

Homme ? Femme ? Il n'y a aujourd'hui sur le Montcalm que des marins qui ont à cœur de servir avec autant d'énergie et de dévouement que leurs anciens (site Intenet du Montcalm).

Ce discours consensuel apparaît cependant en décalage sensible avec les représentations qui persistent au sein de l'équipage très majoritairement masculin. Les chercheurs qui ont étudié la féminisation de ce navire (Dufoulon et al. 1998 ; Trompette et al. 1999) constatent en effet que l'intégration professionnelle des femmes a réussi, notamment à travers la reconnaissance de leurs compétences et de leurs capacités à assumer les contraintes du métier militaire et de la profession de marin. Pour autant, malgré les progrès réalisés dans ce domaine, nous sommes

Anne Schoenfelder et Vincent Tekpor en soient remerciés. Les entretiens ont été réalisés pour l'essentiel en Alsace et en Lorraine. Quatorze entretiens substantiels ont pu être exploités (neuf avec des hommes, âgés de 21 à 51 ans, cinq professionnels et quatre volontaires, et cinq avec des femmes, âgées de 22 à 29 ans, trois professionnelles, dont deux stagiaires et deux volontaires, de huit casernes différentes d'Alsace et de Lorraine. Le corpus des entretiens a été complété par la lecture de plusieurs listes de discussion sur Internet, centrées sur l'entrée des femmes dans les corps de sapeurs-pompiers professionnels (civils et militaires) et volontaires (les intervenants étaient principalement des sapeurs-pompiers, hommes et femmes). 
encore loin de l'avènement de la « femme militaire » ou de la « femme marin ». L'identité masculine est restée au cœur de la représentation identitaire du groupe, bien que l'activité des marins soit de moins en moins associée à l'expression de qualités « viriles » en raison des changements technologiques. Autrement dit, le modèle masculin demeure toujours le référentiel fort, $\mathrm{y}$ compris pour les femmes.

Nous sommes certainement encore plus éloignés de l'avènement de la « femme sapeur-pompier ». Les femmes adhèrent manifestement aussi à un tel modèle masculin. Par contre, elles ont fréquemment à faire face à une hostilité ouverte qui se manifeste souvent collectivement, car elles sont loin d'être acceptées par tous leurs collègues. Au cours des entretiens, très vite les hommes (hormis la hiérarchie) se démarquent de leurs homologues femmes pour les stigmatiser et les inférioriser. Ils n'hésitent pas à affirmer ouvertement que «les femmes n'ont rien à faire dans une caserne » de sapeurs-pompiers ou qu'elles doivent "rester à leur place ». Exclusion, mise à l'écart ou dévalorisation systématique, ouverte ou implicite, y compris éventuellement à travers des attitudes protectrices, telles sont en effet les réactions les plus fréquentes des sapeurs-pompiers hommes à la présence de femmes. À ces réactions négatives s'ajoutent des discours et des attitudes sexistes allant jusqu'au harcèlement sexuel permanent. Ces comportements varient cependant en intensité suivant les femmes visées et les ressources objectives et subjectives qu'elles peuvent mobiliser, comme l'âge, le statut matrimonial, le fait d'être mère ou non, les liens plus ou moins anciens avec le milieu des sapeurs-pompiers, la place dans la hiérarchie, mais aussi selon les manières de réagir des femmes. Ces attitudes défensives mêlent différents registres et discours qui se retrouvent fréquemment dans un même entretien. En reprenant des distinctions classiques en biologie, nous tenterons de démêler les arguments mis en avant par les hommes sapeurs-pompiers, principalement suivant trois niveaux : un niveau éthologique, un niveau écologique et un niveau physiologique ${ }^{14}$.

${ }^{14}$ Cette distinction en trois niveaux (éthologique, écologique et physiologique) m’a été suggérée par Pierre Tripier qui a lu, avec Helena Hirata, une 


\section{Rejeter les femmes pour préserver une " communauté » d'hommes}

«Les femmes n'ont rien à faire dans une caserne »

Au niveau éthologique, les sapeurs-pompiers masculins se présentent comme une "communauté d'hommes " qui cherche à éviter toute intrusion de non-mâles ${ }^{15}$ dans la « Maison des hommes » (Godelier 1982). Les casernes de sapeurs-pompiers sont comparables de ce point de vue aux clubs anglais traditionnels ou aux sociétés de chasseurs (Hell 1994). Tout sera mis en œuvre pour écarter les femmes. Elles sont victimes d'un ostracisme de la part d'une fraction significative des hommes de la caserne :

Ils n'aiment pas les femmes chez les pompiers.

Ils m'ont dit dès le départ que ce ne serait pas facile.

Avant même de se présenter, elles sont déjà stigmatisées. La dévalorisation, la mise à l'écart ou le rejet sont fréquents et ces attitudes s'expriment ouvertement. Dans quelques cas une véritable haine des femmes se manifeste. Les femmes sont confrontées à des hommes qui n'hésitent pas à user de moyens de pression psychologiques qui s’ajoutent alors au stress vécu lors des opérations. Même si les femmes savent d'avance que certains de leurs collègues les mettront mal à l'aise, une telle connaissance, loin de réduire la confusion ressentie, ne fait alors souvent que l'accroître lors des interventions pour lutter contre le feu (Goffman 1974).

Pour justifier leur refus des femmes, une partie des pompiers professionnels invoque leur «faiblesse physique ». L'opposition

première version de ce texte. Je les remercie tous deux pour leurs suggestions stimulantes, mais reste, bien sûr, seul responsable des erreurs ou limites qui subsistent.

${ }^{15}$ Ces « sociétés d’hommes » rejettent fréquemment aussi les non-blancs comme le montre George Orwell dans son roman (2004 [1934]) Burmese days : le Club européen, décrit par l'auteur de 1984, refuse en effet l'adhésion des non-blancs, Birmans ou Indiens, même s'ils exercent des professions prestigieuses, comme celle de médecin. Hormis le témoignage d'un ex-pompier professionnel de Paris, qui a démissionné parce qu'il ne supportait plus l'ambiance machiste et raciste de sa caserne, nous n'avons pas d'autres éléments allant dans le sens d'un rejet ou d'une discrimination vis-à-vis des personnes issues de l'immigration, mais cette question devrait être approfondie lors d'autres enquêtes. 
à leur recrutement s'exprime notamment à travers la mise en cause des barèmes sexués des tests de recrutement :

On fait le même métier, alors pourquoi ce n'est pas le même barème? Donc déjà là ça ne colle pas, hein ?

Un autre :

Si on avait le même barème pour les deux, il y en aurait pratiquement pas qui passeraient. Et là le concours, [au niveau] physique, ils l'ont nettement régressé [sic].

Un autre encore :

Je ne comprends pas pourquoi un mec meilleur ne rentre pas alors que la fille est prise... parce qu'elle a un barème allégé ${ }^{16}$.

Les hommes pompiers considèrent que le métier leur est réservé parce qu'ils sont des hommes. Ils vivent leur identité de pompier comme une identité masculine et, inversement, ils vivent leur identité masculine en tant que sapeur-pompier. De ce point de vue, l'arrivée des femmes dans leur caserne est vécue comme une remise en cause de leur virilité, voire comme une trahison insupportable. Nombreux sont ceux qui s'emploient à justifier leur refus des femmes dans leurs casernes. L'argumentaire fait souvent appel à un registre physiologique :

Déjà une femme, après, elle va se marier elle va avoir des gamins, la logique voudrait... À partir du moment où elle est enceinte, elle n'aura plus le droit d'aller au feu à cause des fumées [...], c'est une personne qui pendant huit mois va être sortie de l'équipe [...], et avec le congé parental après, ça fait que pendant trois ans il y a quelqu'un qui va manquer dans l'équipe ; ça c'est des petits détails.

\footnotetext{
${ }^{16}$ Inversement, des sapeurs-pompiers femmes contestent les nouvelles normes physiques qui s'appliquent désormais aux deux sexes et qui défavorisent de facto les femmes, par exemple les nouvelles normes minimales de taille ont été fixées en 2001 à un mètre soixante pour les hommes comme pour les femmes, abaissant l'ancienne norme masculine et élevant l'ancienne norme féminine. Le résultat ne s'est pas fait attendre, les femmes les plus petites, pourtant admises dans les corps de volontaires, sont désormais exclues des corps de sapeurs-pompiers professionnels : «J'aurais bien aimé être professionnelle mais comme c'est passé à un mètre soixante, je suis trop petite. En tant que volontaire, ils me gardent parce qu'ils m’ont embauchée avant le nouveau texte, mais en tant que professionnelle, je ne peux pas rentrer ».
} 
Ce pompier interrogé exagère les effets de la maternité et dans son esprit le congé " parental », malgré son nom, ne s'adresse qu'aux femmes. Cependant les arguments invoqués semblent être davantage des prétextes puisqu'il sous-entend explicitement que l'essentiel n'est pas là : "ça c'est des petits détails ». Un autre invoque à son tour les maternités, mais aussi le caractère capricieux et inconstant des femmes sur le plan professionnel :

Peut-être qu'elle se lassera du métier, comme on dit, peut-être qu'elle aura deux, trois gamins, puis elle voudra intégrer un service dans un bureau, en journalier, parce qu'elle ne veut plus travailler vingt-quatre heures de garde, puis se ranger comme on dit.

Mais l'argument principal est une fois de plus ailleurs, de l'ordre de l'évidence :

Non je ne pense pas que c'est un métier fait pour les femmes.

Un troisième exprime la même certitude :

Non, je ne vois pas une femme faire ce métier.

Il invoque cette fois une usure prématurée :

Je pense qu'à partir de 35, 40 ans... je vois mal... même 40, 45 ans, je ne dis pas... une femme ne vieillit pas plus vite qu'un homme, mais elle perd plus vite qu'un homme, non?

Un autre prend appui sur l'opinion commune :

Non. À mon avis ce n'est pas un métier pour une femme, et il y a plein de gens qui le disent, ce n'est pas un métier pour une femme.

Une comparaison avec l'armée lui permet de justifier sa position :

Je connais quelqu'un, son fils est à l'armée là, il est parti en Afrique faire des manœuvres, ils avaient une femme dans le peloton, c'est la seule qui traînait et puis ils l'ont dégagée après [sic], parce que physiquement ça ne suivait pas ; et puis les gars l'ont prise à part parce que, eux, ils morflaient, parce qu'elle était toujours derrière.

L'arrivée des femmes dans les casernes de pompiers a été difficile à accepter par les pompiers les plus « anciens ", ceux qui avaient l'habitude de ne côtoyer que du personnel masculin depuis leurs débuts. Jusqu'à une date récente, la politique d’un 
chef de corps était de refuser l'admission de femmes dans sa caserne. Cette situation locale ne s'est débloquée qu'au départ en retraite de ce dernier. Mais les pompiers les plus jeunes, âgés de 20 à 30 ans, partagent très largement ces représentations. Il ne semble pas y avoir de clivage significatif entre les « anciens » et les plus jeunes. Ces derniers estiment comme leurs aînés que leur activité est destinée aux hommes. De ce point de vue, malgré une symbolique très opposée, les jeunes sapeurs-pompiers partagent les mêmes objectifs avec les tagueurs ou les hackers, voire plus largement les informaticiens - milieu professionnel qui en vingt ans a vu la part des étudiantes s'effondrer et passer de $60 \%$ à $10 \%$ (Turkle 1984 ; Auray 2002). On observe en effet dans toutes ces "sociétés de garçons » contemporaines une commune exclusion des femmes. Leur absence permet aux hommes de souder plus facilement le collectif de travail par la communauté d'appartenance de genre. Car ces jeunes construisent leur identité masculine en tant que sapeur-pompier, hacker ou informaticien. L'activité est envisagée en tant qu'activité sexuée, spécifiquement masculine, d'où à la fois le petit nombre de femmes prêtes à s'y investir et l'attitude des membres de ces « sociétés d'hommes » qui refusent l'entrée des femmes. Selon l'interprétation proposée par Danielle ChabaudRychter et Delphine Gardey (2004, p. 231) :

Les relations entre les hommes dans le travail sont fondées sur la compétition, l'évaluation mutuelle des compétences techniques et leur mise en scène dans des épreuves héroïques.

La lutte contre le feu est par excellence une telle activité héroïque que les sapeurs-pompiers masculins n'envisagent pas de partager avec des femmes. Ces dernières sont donc exclues de ces lieux ou de ces activités non seulement parce que les hommes y " "font" de la technique ", mais aussi parce qu' « il s'y fait du masculin ".

L'arrivée des femmes, même peu nombreuses, a perturbé les habitudes de vie des hommes sapeurs-pompiers : d'une certaine manière, ils ont dû se « civiliser » au sens d'Elias :

Une femme chez les pompiers, ce n'est pas évident... avant vous ne faites peut-être pas attention à votre tenue quoi... quand vous êtes en caserne... vous vous laissez aller... alors maintenant... 
Ils n'acceptent que difficilement la remise en cause de leurs normes, notamment en ce qui concerne la tenue vestimentaire ou l'entretien des locaux. Ils contestent aussi l'adaptation des installations existantes, la mise en place de vestiaires, de toilettes spécifiques ou de chambres individuelles.

En caserne professionnelle elles ont leur lit, elles ont leurs toilettes, elles ont... elles ont leur quartier en gros. Mais bon ce n'est pas comme ça qu'on vit en collectivité, c'est tout le monde pareil quoi. Les liens vous ne les formez pas en étant à part... si une femme est toujours à part... vous arrivez à juger quelqu'un si vous le fréquentez, si vous passez du temps avec elle, et pas en étant à part.

L'existence des quartiers réservés aux femmes est très mal vécu par ce sapeur-pompier de 30 ans parce que cet espace particulier permet aux femmes d'échapper un peu au contrôle de la « communauté des hommes ».

Un pompier volontaire d'une quarantaine d'année s'est mis violemment en colère quand je l'interrogeais sur l'arrivée des femmes dans les corps de sapeurs-pompiers :

On n'en veut pas, ce n'est pas fait pour elles. Nous on veut être entre nous. Après les manœuvres, on fait la fête entre nous, on se fait des brochettes dans la forêt, on picole, on se raconte nos trucs. Avec des femmes ce n'est pas pareil. Elles n'ont pas leur place ici.

Dans cette dernière situation, on observe, suivant l'expression utilisée ailleurs par Bertrand Hell (1994) à propos des sociétés de chasseurs, une "prégnance actuelle de schèmes symboliques dérivant d'une vision traditionnelle de l'ordre du monde ».

La caserne de pompiers : un lieu d'expression privilégié de la virilité et de la violence machiste

Le milieu des sapeurs-pompiers est aussi une " communauté d'hommes " au sein de laquelle les interdits verbaux sont tout relatifs. Les "blagues » sexistes rassemblent les hommes qui affirment ainsi leur virilité. Ces « rigolades » collectives ne sont pas sans rapport avec les chahuts des garçons à l'école. Dans tous les cas, il s'agit de prouver sa virilité. Dans les conversations ordinaires, les pompiers masculins ont tendance à tout ramener au sexe, y compris (surtout ?) en présence de femmes. 
Le cul a toujours fait tourner la terre et le sexe fait toujours marrer tout le monde et puis bon, ça détend.

Les rapports sexuels sont mimés de manière grotesque, les organes sexuels sont désignés en termes obscènes, triviaux ou vulgaires.

Dans une caserne, il y a toujours du cul, quoi. En fait, il ne faut pas l'oublier ça.

Les attitudes graveleuses, les approches sexuelles systématiques et les grivoiseries salaces peuvent aller jusqu'au harcèlement permanent, ce dont témoignent différentes plaintes en justice déposées par des femmes sapeurs-pompiers. Réduites à n'être que des objets à plaisanteries sexuelles, elles sont massivement victimes de ces « vannes ». Ces comportements machistes sont comparables à ceux qui ont (ou avaient ${ }^{17}$ ) cours dans des milieux similaires comme l'armée ou la police (Reynaud 1988) mais aussi dans nombre de milieux professionnels civils.

Niveau sexe, quoi, [il siffle], on est focalisé là-dessus. Une nana, dès que tu peux lui faire une remarque là-dessus, ben hop, c'est parti! Elle fait le moindre truc, je ne sais pas moi, comment je pourrais t'expliquer ça ? Le moindre truc, anodin quoi, je veux dire le truc que personne pense, tu as toujours un gars dans le tas [il siffle]... qui fait une remarque dessus.

Ces attitudes sexistes traduisent un refoulement de l'intime. Mais elles sont aussi autant d'agressions ou d'intimidations qui s'expriment la plupart du temps collectivement. Elles contribuent à forger l'unité de la " société » des hommes et à construire la ségrégation entre hommes et femmes, voire l'exclusion de ces dernières, qu'elles soient présentes ou absentes (Elias 1997). Cette violence verbale à caractère sexiste est en effet exercée pour et devant les autres membres du groupe masculin. Les collègues sont alors les témoins et les gardiens de l'ordre viril qui est un impératif pour ces hommes. Cet humour graveleux permet aux hommes d'écraser collectivement, voire de violer, symboliquement la (ou les quelques) femme(s) présente(s), c’est-

\footnotetext{
${ }^{17}$ Il est probable que la situation est pire au sein des casernes de sapeurspompiers en raison du petit nombre de femmes présentes, de plus elles ont moins souvent que dans la police ou l'armée des grades supérieurs à leurs collègues hommes.
} 
à-dire celle(s) qui remet(tent) en question l'existence même de leur «société d'hommes ». Les " cibles » féminines sont alors réduites à n'être qu'un objet sexuel suivant la dichotomie classique de la maman et de la putain. Cependant, les ressources objectives et subjectives que les femmes sapeurs-pompiers mobilisent pour faire face à ces violences entrent aussi en compte. Si une jeune femme refuse fermement ce type de "vanne », les pompiers n'insisteront pas très longtemps et lui témoigneront même un certain respect. Ils sauront qu'elle peut leur tenir tête et éviteront de la « charrier » à l'avenir car ils risqueraient de se faire remettre en place. À l'inverse, celle qui ne réagit pas immédiatement lorsqu'elle est prise comme cible, risque de subir le harcèlement pendant un certain temps :

Si tu vois que c'est une femme qui tout de suite te met une barrière et te dit: "Écoute! Avec moi, tu ne parles pas comme si j'étais la dernière des connasses là ! " [il siffle] Ok, ben je fais : "Ben d'accord». Maintenant si c'est une [femme] qui fait : "Hein! », qui fait que rigoler, tu sais, tu te dis : "Ben attends, celle-là, on va bien la prendre pour une conne».

Ce mépris des femmes qui ne réagissent pas et qui acceptent l'humiliation transparaît aussi dans les propos d'un autre pompier :

Et puis le personnel féminin, s'il le prend bien, il rigole avec nous et puis il en rajoute un peu plus, quoi. Et puis après on se demande qui c'est qui est le plus cochon des deux. Généralement, c'est toujours les femmes mais bon, faut pas le dire [rires].

Pour ces hommes, notamment les plus jeunes, tout se passe cependant comme si ce langage fonctionnait bien comme ressource de réassurance virile dans le cadre collectif de la " société d'hommes », mais qu'il apparaissait disqualifié en dehors de ce cadre. De manière symptomatique, les sapeurs-pompiers interrogés en tête-à-tête par les enquêtrices étaient très gênés quand elles leur demandaient de rapporter de manière précise les propos graveleux ou les blagues salaces.

\section{Délimiter, séparer, démarquer}

À un second niveau, le niveau écologique, les arguments avancés dans les entretiens peuvent s'interpréter comme une stratégie de défense du territoire professionnel des pompiers 
mâles contre «l'espèce femelle » qui tend à l'envahir. Deux types de réactions coexistent à ce niveau. D'une part, on rencontre un discours qui prône la séparation entre deux espèces dont le mélange doit être proscrit. Il s'agit alors d'invoquer la séparation du pur et de l'impur ou du sacré et du profane. Nous sommes alors en présence d'un discours épidémiologique classique (Boulding 1962) qui rejoint en partie ce qui se joue au niveau éthologique que nous venons d'examiner. Il en résulte notamment une division des tâches sexuée conforme aux stéréotypes sociaux, division des tâches qui écarte symboliquement les femmes de la lutte contre le feu et les confine dans des activités dans lesquelles elles peuvent mettre en œuvre leurs compétences «naturelles».

«Une femme sur un incendie, elle n'a rien à y faire »

Que les femmes soient employées comme standardistes, secrétaires, voire conductrices d'ambulances ou secouristes passe encore, mais l'idée qu'elles puissent aller au feu fait sortir de leurs gonds plusieurs des pompiers interrogés :

Une femme sur un incendie, elle n'a rien à y faire.

Ce n'est pas pour ça que la femme est faite.

Oh non! Non, non. Ça, c'est... ce n'est rien de bon.

En somme, comme le note encore un autre pompier :

Il y a certaines choses qu'il faut peut-être laisser aux hommes, quoi.

Quand l'argumentation ne se réduit pas à l'ordre de l'évidence, le refus de laisser les femmes aller au-devant du feu s'appuie sur trois registres : la difficulté physique des tâches à accomplir, le danger inhérent à la situation et la peur engendrée par le danger. Celle-ci leur est imputée comme une caractéristique "naturelle » de leur féminité. De même, leurs manifestations de souffrance (peur, doute, interrogations morales, compassion...) sont immédiatement interprétées comme étant la marque de leur infériorité « naturelle ». L'adhésion des hommes sapeurs-pompiers aux critères standards de la virilité leur permet en effet, comme les travailleurs du bâtiment ou les sous-traitants du nucléaire, de surmonter le danger et la peur (Pezé 2002 ; Molinier 2004). Ils imaginent difficilement une telle adhésion de la part de femmes, car pour cela elles doivent, comme le dit 
explicitement l'un des pompiers, "laisser leur sensibilité au vestiaire ». On retrouve ici les tensions déjà relevées pour d'autres professions :

Pour les femmes, il existe une forte contradiction entre la construction de la féminité et l'intégration dans le monde du travail. [...] Les femmes qui veulent faire une carrière valorisée doivent adhérer au système de défense viril, en méprisant du même coup leur propre sexe (Molinier, Welzer-Lang 2004, p. 80).

Les femmes sont confrontées en permanence au jugement dépréciatif et aux commentaires dévalorisants de leurs collègues masculins. Pour être acceptées, elles doivent davantage faire leurs preuves que leurs homologues, s'endurcir et adhérer aux critères de virilité. Un homme explique :

Si une femme vient, elle fait tout, je veux dire qu'elle n'est pas en train de pleurer là, j'ai mal là...

Une jeune femme sapeur-pompier confirme :

Il faut avoir du courage et ne pas être sensible parce que les hommes au début... c'est quand même macho, quoi, et je veux dire, il ne faut pas se laisser abattre parce que si on n'est pas forte de caractère, je pense qu'on se laisse facilement... abattre moralement parce que... tu vas te dire : "Ouais, c'est vrai, lui, il me dit qu'une femme, ça n'a rien à faire là et puis il a peutêtre raison. " Si on est trop sensible, on pensera à ça, nous par contre, si on a vraiment envie de le faire, on y arrivera.

Dans ces conditions, on comprendra que les femmes qui entrent dans ce type de profession (ou d'activité) ont le choix entre adhérer aux représentations masculines, voire machistes, du milieu et du métier ou renoncer. Une jeune femme, sapeurpompier volontaire, précise :

Les filles qui étaient déjà là jusqu'à présent, elles sont toutes parties au bout de même pas un an, voire un mois après déjà.

Suivant les modèles stéréotypés, les hommes disposent de la force physique, de l'autorité et, plus largement, de tout un ensemble de dispositions corporelles viriles naturalisées. Ces « qualités » leur permettent de faire face à la dangerosité et aux risques de l'emploi. Mais en même temps les hommes tendent à monopoliser les tâches les plus valorisées, en particulier, en ce qui concerne les pompiers, les premiers secours en cas 
d'incendie. A contrario, l'image des femmes qui est mobilisée par leurs collègues est souvent celle, caricaturale, d' " une taille 36 » qui fait « 50 kilos tout mouillée ». L'argument ultime consiste alors à dire : "Mais physiquement, elles ne sont pas illimitées » [sic]... comme si c'était le cas des hommes.

Pour se valoriser et confirmer leur supposée supériorité, les sapeurs-pompiers manifestent aussi des attitudes protectrices :

Une femme n'a pas toujours la même force physique. On peut très bien l'aider quoi.

Mais cette aide est loin d'être désintéressée :

Le problème, c'est qu'on est encore trop gentils, on fait tout pour elles, et ça, ça ne va pas. C'est là où ça coince en fait.

Je ne vais pas être nul, lui demander de porter des trucs où elle va commencer à se casser le dos, ben je porte pour elle, et puis c'est réglé. Maintenant, il faut qu'elle reste à sa place.

À partir du moment où elles bénéficient de leur aide, les femmes sont «cataloguées » par les hommes, c'est-à-dire qu'elles sont considérées comme inférieures et, à partir de là, comme ne pouvant pas ou plus se débrouiller seules. Cette " protection » apportée par leurs collègues masculins se double fréquemment de sollicitations sexuelles :

Ils sont assez protecteurs, un petit peu galants aussi, [mais] au boulot je ne cherche pas cette galanterie, je cherche plutôt l'égalité.

Ils pensaient que j'étais venue chez les pompiers pour me trouver un copain, quoi, alors que ce n'est pas du tout le cas. Mais eux, ils pensent toujours à ça, c'est... ils ne voient que ça.

L'une des femmes sapeurs-pompiers interrogées semble intérioriser cette infériorisation des femmes sur le plan physique :

$A u$ feu, il y a plus de danger, on a un matériel qui pèse presque vingt kilos sur le dos, donc quand on a plus d'un tiers de son poids sur le dos, c'est plus difficile pour une femme, je pense, que pour un homme [...]. C'est quand même assez physique, il ne faut pas avoir peur d'y aller. C'est pour ça qu'il y en a qui pensent que la femme pompier ne devrait faire que de l'ambulance... et pas le feu. [...] Un gros incendie, avec des personnes inconscientes au deuxième étage, et qu'il faut évacuer, donc il faut faire des dégagements d'urgence, c'est vrai que si la personne fait 
quatre-vingts ou cent kilos, je vais certainement avoir quelques soucis à... porter sur le dos, même avec la manière pompier, qui pourtant permet beaucoup de choses, je pense que j'aurai des problèmes, donc c'est vrai que je suis censée être capable de faire, je le suis peut-être je n'en sais rien, je n'ai jamais eu à le tester heureusement...

Mais, après avoir souligné dans le passage cité les difficultés rencontrées, elle ajoute, cependant, un argument décisif qui rompt avec la vision viriliste et individualiste des tâches du pompier, en particulier lors des interventions sur les incendies :

Il ne faut pas oublier non plus que le travail de pompier c'est un travail d'équipe.

Il est frappant de constater que cette dimension collective de l'action des pompiers n'est jamais mentionnée par les hommes, sauf quand ils font partie du commandement, et qu'ils jaugent et jugent systématiquement les femmes isolément, en les retirant de ce collectif.

«Les femmes sont meilleures en secourisme »

Le pendant du discours épidémiologique qui vise à écarter les femmes de la lutte contre le feu, c'est un discours de complémentarité qui consiste à accepter les femmes parmi les sapeurspompiers à condition qu'elles restent à leur «juste place ». Autrement dit, elles sont acceptées si elles ne se mêlent pas des activités considérées comme masculines et si elles assurent les tâches pour lesquelles elles sont reconnues comme compétentes. L'introduction de la mixité dans les corps de sapeurs-pompiers s'accompagne alors du développement d'une division sexuée des tâches sur les plans formel et informel. Les femmes tendent à occuper de facto des postes différents de ceux des hommes. La division du travail qui tend à s'instaurer est alors en phase avec les stéréotypes sociaux de sexe fortement enracinés dans ce milieu masculin. Les domaines d'emploi et de travail tendent à se spécialiser. Le contenu des emplois va se différencier et la division du travail traditionnelle entre les sexes se reproduire. Les savoirs et savoir-faire acquis dans la socialisation sont mobilisés pour justifier la sexuation des tâches professionnelles. Deux versions du métier tendent alors à se développer, comme dans l'exemple classique de l'imprimerie analysé par Cynthia 
Cockburn (1983). À côté de la version masculine, les femmes se voient attribuer des compétences relationnelles, faites de convivialité, d'écoute, d'accueil, d'empathie, de séduction, de douceur, de patience, de dialogue. Les pratiques des femmes sapeurs-pompiers tendent à s'adapter aux stéréotypes sociaux de sexe, par exemple s'occuper des premiers soins aux personnes, tout en pouvant dans certains cas y contrevenir.

Un troisième niveau argumentatif, le niveau physiologique, vient alors compléter ou spécifier le discours de complémentarité précédent : il est explicitement fait appel aux « qualités » de chaque sexe considérées comme " naturelles ». La présence des femmes au feu est particulièrement stigmatisée, elles seraient incapables de prêter main-forte à leurs collègues masculins en détresse, leurs compétences professionnelles sont niées, seules leurs caractéristiques féminines "naturelles » sont mises en valeur. Les sapeurs-pompiers masculins multiplient les considérations à ce sujet :

Le secours à victimes ça c'est un truc où, je l'ai toujours dit haut et fort, c'est clair que là les femmes ont peut-être plus de... de doigté que les hommes quoi, elles ont peut-être plus de facilité à rassurer les gens.

Le contact passe plus ; parce qu'elles ont un feeling [...], une femme pourra avoir plus d'informations, pour savoir le pourquoi du comment. Ce qu'il prend comme médocs ${ }^{18}$, pourquoi il est dans cet état, c'est plus facile de se confier à une femme que se confier à un homme.

Elles ont des qualités d'approche de la victime que les hommes ont moins.

Maintenant, elles ont d'autres avantages, c'est vrai qu'au niveau de l'ambulance, elles sont beaucoup plus douces et donc là, à ce niveau-là, c'est vrai qu'on a plus à... qu'on a pas mal à apprendre d'elles.

Bon c'est quand même plus... plus rassurant pour une victime, bon, je pense hein, de se faire aborder par une fille qui a déjà une voix plus douce que par trois gars d'un mètre quatre-vingt-dix...

Un gars n'aura pas autant de tact à parler...

\footnotetext{
${ }^{18}$ Médicaments.
} 
Les femmes... il y en a qui, côté relationnel justement sont bien, ça apaise pour certaines interventions, le fait qu'il y a une femme avec, ça apaise certainement, aussi bien le personnel de notre côté que du côté de la victime, parce qu'un gars de notre côté va aussi se retenir de s'emporter vis-à-vis d'un gars qui est prêt à tout casser, le gars qui veut tout casser s'il voit qu'il y a une femme, c'est toujours pareil... moi je pense que j'aurais du mal à taper une femme.

En somme, on l'a compris, si elles restent à leur place, les femmes « apaiseraient » aussi bien les victimes d'accidents ou d'incidents que... leurs collègues masculins...

Enfin, se mettant à la place d'un homme secouru, un autre sapeur-pompier illustre lui aussi la puissance des stéréotypes :

Avec une femme, il y a toujours cette retenue, je vais pas cogner sur une femme parce que c'est une femme, c'est vrai, en plus parce que c'est une femme, elle est mignonne, je ne vais pas aller avec elle à l'hôpital.

Les femmes sapeurs-pompiers ne sont pas en reste :

On a une approche différente des gens, on arrive plus à rassurer les gens qu'un homme, ça passe beaucoup mieux en fait avec une femme qu'avec un homme.

Je crois qu'on a un peu plus de patience, on a peut-être une manière de pouvoir plus tempérer certaines ardeurs de comportements ou certaines violences verbales, voire physiques, on arrive parfois à tempérer les choses et à faire que les choses ne s'enveniment pas.

Parce qu'on a une autre écoute, une meilleure écoute ou... une façon plus douce.

Résultat : les femmes sont affectées au secourisme, notamment au secours à victime. L'une des femmes interrogées le formule à sa manière :

Les femmes sont plus, comment dire ? attitrées [sic] au secourisme.

Ce que confirme une autre :

Quasiment dans tous les centres de secours les femmes sont affectées aux VSAV ${ }^{19}$.

${ }^{19}$ Véhicules de secours aux victimes. 
Les femmes auraient «des aptitudes plus... prononcées pour le secourisme [...], c'est comme ça ». "Une femme, on l'a toujours dit et je pense qu'on le répètera toujours, les femmes sont meilleures en secourisme ». "Le côté féminin en fait » sera invoqué tant par les hommes que par les femmes : la douceur, la patience, le "feeling ", l'écoute, le dialogue plus facile, la confiance qu'elles inspirent, les «qualités d'approche » de la victime, le sourire, « rassurer les personnes, les tranquilliser». On retrouve ici l’idée que :

Les femmes, à la différence des hommes, ne construiraient pas leur compétences, mais disposeraient d'un fond naturel de dons et de qualités féminines (dextérité, minutie, patience, empathie, etc.) [et de ce fait] les prestations féminines sont considérées comme normales de la part d'une femme (Molinier, WelzerLang 2004, p. 79-80).

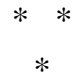

Les jeunes femmes qui veulent s'engager et s'investir dans un corps de sapeurs-pompiers entrent donc dans un monde faiblement féminisé. La part des femmes est tellement réduite qu'elles doivent le plus souvent affronter seules ou en tout petit nombre un univers hostile. Elles ont à faire face à de multiples réactions défensives de la part de leurs collègues masculins. Elles seront jaugées, évaluées, «testées », aussi bien sur le plan mental que physique. Durant les manœuvres, les entraînements ou les interventions, les femmes sapeurs-pompiers doivent endurer la souffrance liée aux exercices physiques ou aux situations de danger en subissant en outre les regards, les attitudes ou les commentaires de leurs collègues qui les mettent à l'épreuve, bien davantage que les nouveaux arrivants masculins. Les pressions de diverses natures ne manquent pas. À la question : "Quand il y a une nouvelle femme pompier, tu réagis comment ? ", un jeune pompier répond :

Test. Test. Je ne vais pas mettre ma confiance en des gens sur un coup de tête.

Un autre :

Si tu n'es pas capable de faire comme les hommes, ben tu n'es pas capable d'être pompier. 


\section{Un troisième :}

Si elle vient et que les premières fois quand elle se fait bizuter, elle commence à râler et à aller se plaindre, ben là c'est fini, non mais là, elle a toute la caserne à dos, ce n'est même plus la peine qu'elle vienne. Là, elle va se faire tuer.

Résultat, pour être à la hauteur, tenir tête, ne pas craquer, ne pas être discréditées par leurs collègues, elles doivent en faire davantage :

Les femmes doivent faire plus d'efforts encore pour convaincre tout le monde (jeune pompier fraîchement recruté).

Je prenais des gardes même en plus. [...] J'y suis arrivée plus vite... pour leur prouver qu'une femme peut aussi arriver à faire ce qu'ils font eux (jeune femme sapeur-pompier).

Dans cet univers, fortement masculinisé, davantage encore que dans l'armée ou la police, la répartition des tâches est toujours structurée suivant la hiérarchie des rapports sociaux de sexe. Le monde des sapeurs-pompiers permet in fine de saisir la puissance des stéréotypes de sexe presque à l'état brut et de mesurer à quel point l'entrée des femmes dans un milieu d'hommes ne va vraiment pas de soi.

\section{Références}

Appert Monique, Grozelier Anne-Marie, Baron Cécile et al. (1983). « Diversification de l'emploi féminin. Insertion professionnelle des femmes dans des métiers traditionnellement masculins ». Dossier CEE, $\mathrm{n}^{\circ} 8$.

Auray Nicole (2002). " Sociabilité informatique et différence sexuelle ». In Chabaud-Rychter Danielle, Gardey Delphine (eds). L'engendrement des choses. Des hommes, des femmes et des techniques. Paris, Archives contemporaines.

Bihr Alain, Pfefferkorn Roland (1996). Hommes-Femmes, l'introuvable égalité : école, travail, couple, espace public. Paris, L’Atelier «Points d'appui ».

- (2002). Hommes-Femmes, quelle égalité ? Paris, L’Atelier.

Bouchard Marie-Amélie (1996). «La féminisation des écoles d'officiers : Écoles de l'air, École spéciale militaire, École navale ». Paris, C2SD.

Boulding Kenneth (1962). Conflict and Defense. A General Theory. New York, Harper \& Row. 
Chabaud-Rychter Danielle, Gardey Delphine (2004). « Techniques et genre ». In Hirata Helena et al. Dictionnaire critique du féminisme. Paris, PUF « Politique d'aujourd'hui » [1 ${ }^{\text {re }}$ éd. 2000].

Cockburn Cynthia (1983). Brothers. Male Dominance and Technological Change. London, Pluto Press.

Criqui Étienne (1992). «Le canton en héritage ». In Patriat Claude, Parodi Jean-Luc (eds). L'hérédité en politique. Paris, Economica.

Devreux Anne-Marie (1997). " Des appelés, des armes et des femmes : l'apprentissage de la violence masculine à l'armée ». Nouvelles questions féministes, vol. 18, n 3-4, novembre.

Dufoulon Serge, Saglio Jean, Trompette Pascale (1998). La différence perdue : la féminisation de l'équipage du Montcalm. Paris, C2SD «Les Documents du C2SD ».

Elias Norbert (1997). Les logiques de l'exclusion. Paris, Fayard.

Fortino Sabine (1999). "De la ségrégation sexuelle des postes à la mixité au travail : étude d'un processus ». Sociologie du travail, vol. $41, \mathrm{n}^{\circ} 4$.

- (2002). La mixité au travail. Paris, La Dispute « Le genre du monde ».

Gadrey Nicole (2001). Travail et genre. Approches croisées. Paris, L'Harmattan « Logiques sociales ».

Géo (2002). « Pompiers du monde », hors-série, 15 mai.

Godelier Maurice (1982). La production des grands hommes : pouvoir et domination masculine chez les Baruya de Nouvelle-Guinée. Paris, Fayard «L'espace du politique ».

Goffman Erving (1974). Les rites d'interaction. Paris, Minuit.

- (2002). L'arrangement des sexes. Paris, La Dispute « Le genre du monde ».

Hell Bertrand (1994). Le sang noir. Chasse et mythe du sauvage en Europe. Paris, Flammarion.

Hervieu-Léger Danièle (1999). Le pèlerin et le converti. La religion en mouvement. Paris, Flammarion.

Hirata Helena, Senotier Danièle (eds) (1996). Femmes et partage du travail. Paris, Syros « Alternatives sociologiques ».

Kergoat Danièle et al. (1992). Les infirmières et leur coordination. 1988-1989. Paris, Lamarre.

Kihl Jean-Paul (2000). " Les sapeurs-pompiers en France ». Regards sur l'actualité, vol. 259, mars.

Lussier Hubert (1987). Les sapeurs-pompiers au XIX $X^{e}$ siècle. Associations volontaires en milieu populaire. Paris, ARF et L'Harmattan. 
Maruani Margaret (ed) (1998). Les nouvelles frontières de l'inégalité. Hommes et femmes sur le marché du travail. Paris, La Découverte.

Molinier Pascale (2004). « Dépression sous les neutrons : une enquête dans l'industrie de process ». Cahiers du genre, $n^{\circ} 36$ « Les résistances des hommes au changement » (Anne-Marie Devreux, ed).

Molinier Pascale, Welzer-Lang Daniel (2004). « Féminité, masculinité, virilité ». In Hirata Helena et al. Dictionnaire critique du féminisme. Paris, PUF « Politique d'aujourd'hui » [1 ${ }^{\text {re }}$ éd 2000].

Monrique Michèle (2004). " Place des femmes dans la professionnalisation des armées ». Avis et rapports du Conseil économique et social, $\mathrm{n}^{\circ} 20$, juillet.

Orwell George (2004 [1934]). Une histoire birmane. Paris, Ivrea [éd. originale (1934). Burmese days. New York, Harpers \& Brothers].

Perrot Michelle (1998). Les femmes ou les silences de l'histoire. Paris, Flammarion.

Pezé Marie (2002). Le deuxième corps. Paris, La Dispute « Le genre du monde ».

Pruvost Geneviève (2005). L'accès des femmes à la violence légale. La féminisation de la police (1935-2005). Thèse sous la direction de Rose-Marie Lagrave, CEMS-EHESS.

Retière Jean-Noël (1994). « Être sapeur-pompier volontaire : du dévouement à la compétence ». Genèses, n 16 , juin.

Reynaud Emmanuel (1988). Les femmes, la violence et l'armée. Essai sur la féminisation des armées. Paris, Fondation pour la Défense nationale \& La Documentation française.

Sorin Katia (2003). Femmes en armes, une place introuvable ? Le cas de la féminisation des armées françaises. Paris, L’Harmattan "Logiques sociales».

Trompette Pascale, Saglio Jean, Dufoulon Serge (1999). « Métier militaire et identité féminine. La féminisation de la frégate Montcalm ». Les Champs de Mars, $n^{\circ}$ 5, avril.

Turkle Sherry (1984). The Second Self. Computers and the Human Spirit. New York, Simon \& Schuster [trad. française par Claire Demange (1986). Les enfants de l'ordinateur. Paris, Denoël]. 